\title{
Polarization Dynamics of Current-Modulated Vertical-Cavity Surface-Emitting Lasers
}

\author{
Cristina Masoller, Maria Susana Torre, and K. Alan Shore, Senior Member, IEEE
}

\begin{abstract}
We study the dynamics of vertical-cavity surface-emitting lasers (VCSELs) with direct current modulation in the framework of a model for index-guided VCSELs that takes into account two orthogonal linear polarizations. We analyze the effect of current modulation near the polarization switching (PS) of type I, from the high to the low frequency polarization, and near the PS of type II, from the low to the high frequency polarization. We find that the oscillations of the total power are as those of a single-mode laser, unaffected by the underlaying polarization coexistence or polarization competition. We also study the small-signal modulation response in the Fourier domain, for modulation dc values near the PS point. Close to type I PS the response of the total power as well as the response of the orthogonal polarizations has the same functional dependency on the modulation frequency, and can be fitted by the response function of a single-mode laser. Close to type II PS, polarization competition is a significant process at low modulation frequencies. The polarization-resolved modulation response displays features at low frequencies that are not present in the response of the total power, which is as that of a single-mode laser. The dynamics becomes increasingly complex as the modulation amplitude grows, and there is multistability of solutions.
\end{abstract}

Index Terms-Current modulation, polarization switching, vertical-cavity surface-emitting lasers (VCSELs).

\section{INTRODUCTION}

$\mathbf{V}$ ERTICAL-CAVITY surface-emitting lasers (VCSELs) are now widely used as low-cost coherent sources in optical communication systems. They offer many advantages over conventional, edge-emitting lasers, such as low threshold current, small volume, high efficiency, single-longitudinal-mode operation, and a circular output beam. However, in VCSELs with circular transverse geometry, the orientation of the polarization of the emitted light is not fixed by geometrical

Manuscript received April 19, 2007; revised July 5, 2007. This research was supported in part by the U.S. Air Force Office of Scientific Research under Grant FA9550-07-1-0238. The work of C. Masoller was supported in part by the "Ramon y Cajal" Program (Spain) and the Spanish Ministerio de Educacion y Ciencia through Project FIS2005-07931-C03-03. The work of M. S. Torre was supported in part by the CONICET Grant PIP 6474, in part by the FONCyT Grant 3/09598, Argentina, and in part by the Royal Society Argentina-U.K. joint project.

C. Masoller is with the Departament de Fisica i Enginyeria Nuclear, Universitat Politecnica de Catalunya, E-08222 Terrassa, Spain (e-mail:cristina.masoller@upc.edu).

M. S. Torre is with the Instituto de Física Arroyo Seco, Universidad Nacional del Centro de la Provincia de Buenos Aires, Pinto 399 (7000) Tandil, Argentina (e-mail:marita@exa.unicen.edu.ar).

K. A. Shore is with the Schools of Electronics and Computer Science, University of Wales, Bangor LL57 1UT, U.K. (e-mail: alan@ informatics.bangor.ac. uk).

Color versions of one or more of the figures in this paper are available online at http://ieeexplore.iee.org.

Digital Object Identifier 10.1109/JQE.2007.907237 constraints (as it is in edge-emitters). When VCSELs start to lase they usually emit a linear polarization, with the vector field oriented along one of the two orthogonal directions associated with the crystal axes. As the injection current is increased, a polarization switching (PS) to the orthogonal linear state often occurs. The PS phenomenon is often accompanied by complex polarization dynamics including polarization coexistence (simultaneous emission on two orthogonal linear polarizations with different emission frequencies), polarization competition (noise-induced hopping between two orthogonal linear polarizations with different emission frequencies), and the emission of elliptically polarized light (simultaneous emission on two orthogonal linear polarizations with the same emission frequency) [1]-[14]. Polarization mode dispersion is a significant issue in optical fiber communications systems (see, for example, [15] for a recent special issue devoted to polarization effects in fiber-optic networks). The polarization instabilities of VCSELs need to be taken into account for the use of these devices as coherent light sources in such communications systems, and several authors have proposed methods to control the polarization state of the emitted light [16]-[19].

For high bit-rate communication systems, lasers with a large modulation bandwidth are required. The modulation bandwidth of a laser is mainly determined by the relaxation oscillation frequency of the laser, beyond which the modulation response decreases rapidly. The modulation response of VCSELs has received a lot of attention [20]-[30], but few studies have focused on the impact of polarization instabilities.

Verschaffelt et al. [31] studied experimentally the VCSEL response near the PS. By modulating the bias current around the PS point and measuring the critical modulation amplitude necessary to force polarization switching, as a function of the modulation frequency, they obtained a polarization modulation frequency response (PMFR) that showed the same cut-off frequency as the thermal frequency response, suggesting the importance of thermal effects. In a follow up study Verschaffelt $e t$ al. [32] compared the PMFR of gain-guided and index-guided VCSELs, and found different dynamic behavior for the different VCSEL types: while thermal effects only play a minor role in the PS of index-guided VCSELs, they seem to play a key role in the PS of gain-guided VCSELs.

Valle et al. [33] studied numerically the response of index-guided VCSELs to high-frequency current modulation and found chaotic behavior in the regime of multi-transverse-mode emission, but not in the single-mode regime, where only periodic behaviors -with coexistence of periodic solutionswere found. Switching between the different solutions was possible by the external injection of optical pulses. While 
the model used in [33] takes into account key mechanisms determining transverse-mode competition, such as spatial hole burning, wave-guiding effects, and carrier diffusion, it does not consider the polarization of the transverse modes. A study of current-modulated VCSELs taking into account polarization effects but neglecting transverse effects was performed by Sciamanna et al. [34]. The intensities of the two polarizations were found to exhibit periodic or chaotic regimes with combination of in-phase and out-of-phase dynamics at two different time scales, associated with the modulation frequency and the relaxation oscillation frequency.

We have recently studied numerically the interplay of current modulation and weak optical feedback [35], using the model for index-guided VCSEL that takes into account transverse but not polarization effects. For increasing modulation amplitude we found a transition from a regime governed by the optical feedback to a regime where both feedback and modulation contribute, to a regime governed by the current modulation. Spectral signatures of these regimes were observed experimentally by Hong et al. [36], who also characterized a resonance that depends on the external cavity length.

So far, to the best of our knowledge, no study has addressed the impact of polarization competition and transverse effects on the VCSEL modulation characteristics. In this work we study the dynamics of current-modulated VCSELs, including both transverse and polarization effects. We use the framework of the spin-flip model [4] to take into account the polarization, and the index-guided approximation to include transverse effects [3], [5]. The transverse optical profiles and modal frequencies are determined by the built-in refractive index distribution, thus allowing a description in terms of modal amplitudes. For the cylindrical transverse section of VCSELs, and assuming operation near threshold with fundamental-mode emission in both polarizations, the appropriate optical profile is the $L P_{01}$ mode. Birefringence is taken into account assuming that the two orthogonal polarizations have slightly different refractive indexes in the core region of the waveguide. Thus, birefringence results not only in a frequency split between the two polarizations, but also, in differently confined transverse profiles, $L P_{01}^{x}$ and $L P_{01}^{y}$, and thus, in different modal gains.

To investigate the impact of transverse and polarization effects on the dynamics of current modulated VCSELs, we performed numerical simulations of the model equations focusing on the region of injection currents where the PS occurs. We studied the two types of PS, from the high to the low frequency polarization (commonly referred to as type I) and from the low to the high frequency polarization (referred to as type II) [12]. We found that the small-signal polarization-resolved modulation response differs for type I and type II PS. Close to type I PS the response of the total power as well as the response of the orthogonal polarizations has the same functional dependency on the modulation frequency, and can be fitted by the response function of a single-mode laser. Close to type II PS, polarization competition is a significant process at low modulation frequencies, and the polarization-resolved modulation response displays features at low frequencies that are not present in the response of the total power, which is as that of a single-mode laser. For large modulation amplitude we find complex pulsing behavior, including polarization competition and polarization coexistence.

This paper is organized as follows. Section II presents the model, Section III presents the numerical results and Section IV presents a summary and the conclusions.

\section{MODEL}

Full details of the model are given in [5], [37] and thus here we simply highlight its main features. The equations for the two linearly polarized slowly-varying complex amplitudes of the optical field, $E_{x}$ and $E_{y}$, the total carrier density $N$, and the population difference $n$, between the carrier densities with positive and negative spin values, are

$$
\begin{aligned}
\frac{d E_{x}}{d t}= & k(1+i \alpha)\left[\left(G_{x}-1\right) E_{x}+i g_{x y} E_{y}\right] \\
& -\left(\gamma_{a}+i \gamma_{p}\right) E_{x}+\sqrt{\beta_{s p}} \xi_{x}(t) \\
\frac{d E_{y}}{d t}= & k(1+i \alpha)\left[\left(G_{y}-1\right) E_{y}-i g_{x y} E_{x}\right] \\
& +\left(\gamma_{a}+i \gamma_{p}\right) E_{y}+\sqrt{\beta_{s p}} \xi_{y}(t) \\
\frac{\partial N}{\partial t}= & -\gamma_{N}\left[-\mu+N\left(1+\left|E_{x}\right|^{2} \psi_{x}^{2}+\left|E_{y}\right|^{2} \psi_{y}^{2}\right)\right. \\
& \left.+i n\left(E_{y} E_{x}^{*}-E_{x} E_{y}^{*}\right) \psi_{x} \psi_{y}\right] \\
& +\frac{D\left(\frac{1}{r}\right) \partial}{\partial r}\left[\frac{\left(\frac{1}{r}\right) \partial N}{\partial r}\right] \\
\frac{\partial n}{\partial t}= & -\gamma_{s} n-\gamma_{N}\left[n\left(\left|E_{x}\right|^{2} \psi_{x}^{2}+\left|E_{y}\right|^{2} \psi_{y}^{2}\right)\right. \\
& \left.+i N\left(E_{y} E_{x}^{*}-E_{x} E_{y}^{*}\right) \psi_{x} \psi_{y}\right] \\
& +\frac{D\left(\frac{1}{r}\right) \partial}{\partial r}\left[\frac{\left(\frac{1}{r}\right) \partial n}{\partial r}\right] .
\end{aligned}
$$

Here, $k$ is the field decay rate, $\gamma_{N}$ is the carrier decay rate, $\gamma_{s}$ is the decay rate which accounts for the mixing of the populations with different spins due to spin-flip relaxation processes. $\alpha$ is the linewidth enhancement factor, $D$ is the diffusion coefficient, $\beta_{s p}$ is the spontaneous emission strength, $\xi_{x, y}$ are uncorrelated Gaussian white noises with zero mean.

The gain terms are given by the overlap of the transverse profiles with the carrier density

$$
\begin{aligned}
G_{x, y}(t) & =\int_{0}^{\infty} N(r, t) \psi_{x, y}^{2}(r) r d r \\
g_{x y}(t) & =\int_{0}^{\infty} n(r, t) \psi_{x}(r) \psi_{y}(r) r d r .
\end{aligned}
$$

It is assumed that the laser emits the fundamental transverse mode in both polarizations, $\psi_{x}=L P_{01}^{x}$ and $\psi_{y}=L P_{01}^{y}$, and the profiles are normalized such that $\int_{0}^{\infty} \psi_{x, y}^{2}(r) r d r=1$.

Birefringence is taken into account assuming that the refractive index in the core region in the $x$ direction $n_{x}^{\text {core }}$ is different from that in the $y$ direction $n_{y}^{\text {core }}$, while in the cladding region $n^{\text {clad }}$ is isotropic [5]

$$
\begin{aligned}
& n_{x, y}(r)=n_{x, y}^{\text {core }} \text { for } r \leq a, \\
& n_{x, y}(r)=n^{\text {clad }} \text { for } r>a .
\end{aligned}
$$

Here $a$ is the radius of the core region of the waveguide. As a consequence of birefringence, the transverse profiles $\psi_{x}$ and 
$\psi_{y}$ are different, and thus, the two polarizations have different modal gains, given by (5).

The parameter $\gamma_{a}$ is the dichroism parameter that accounts for an external gain or loss anisotropy, which is not due to the different overlap of the transverse profiles with the active region. In the absence of transverse inhomogeneities, when $\gamma_{a}>0$ the laser begins to lase on the $y$ polarization because it has the lower threshold. However, when transverse inhomogeneities are taken into account, the polarization selected at threshold is determined not only by $\gamma_{a}$ but also by the overlap of the mode profiles with the gain region.

The parameter $\gamma_{p}$ accounts for the frequency splitting of the two polarizations and is determined by eigenvalue equations involving $n_{x, y}^{\text {core }}$ and $\left.n^{\text {clad }} \cdot \gamma_{p}=\left(\omega_{y}-\omega_{x}\right) / 2\right)$, where $\omega_{y}-\omega_{x}=$ $c\left(k_{o y}-k_{O x}\right) . k_{o y}$ and $k_{O x}$ are calculated from the eigenvalue (6) and (7) of [37]. When $n_{x}^{\text {core }}>n_{y}^{\text {core }}\left(n_{x}^{\text {core }}<n_{y}^{\text {core }}\right)$ the $x$ polarization has better (worst) transverse confinement and has the lower (higher) emission frequency, and thus, $\gamma_{p}>0\left(\gamma_{p}>0\right)$.

The parameter $\mu$ is the normalized injection current: $\mu_{\mathrm{th}}=1$ in the absence of anisotropies and transverse inhomogeneities. The injection is assumed to be uniform in the core region, and zero outside:

$$
\begin{aligned}
& \mu(r, t)=j(t) \quad \text { for } r \leq a \\
& \mu(r, t)=0 \quad \text { for } r>a .
\end{aligned}
$$

The modulated current is

$$
j(t)=j_{\mathrm{dc}}+A_{m} \cos \left(2 \pi f_{m} t\right)
$$

where $j_{\mathrm{dc}}$ is the dc-value, $A_{m}$ is the modulation depth and $f_{m}$ is the modulation frequency.

\section{RESULTS}

We simulated the model equations with typical parameters indicated in Table I [4], [5], [37]. To study the polarization competition induced by the interplay of current modulation, spatial hole burning and waveguiding effects, we vary the modulation parameters, $j_{\mathrm{dc}}, A_{m}, f_{m}$, the carrier diffusion rate, $D$, and the refractive index of the $x$ polarization in the active region, $n_{x}^{\text {core }}$. Since the characteristic equation that governs the linear stability of the $x$ and $y$ polarizations remains unchanged if: 1) the polarizations are interchanged $(x \rightarrow y, y \rightarrow x)$ and 2) the signs of both dichroism and birefringence parameters are changed $\left(\gamma_{a} \rightarrow-\gamma_{a}\right.$ and $\left.\gamma_{p} \rightarrow-\gamma_{p}\right)$ [4], it is enough to consider a situation in which the $x$ polarization is better confined than the $y$ polarization $\left(n_{x}^{\text {core }}>n_{y}^{\text {core }}\right.$ and thus $\gamma_{p}>0$ ). Changing the sign of both $\gamma_{p}$ and $\gamma_{a}$ corresponds to the same physical situation, but for fixed sign of $\gamma_{p}$, different signs of $\gamma_{a}$ correspond to different physical situations.

\section{A. Small-Amplitude Modulation Near Type II PS}

We begin by considering a situation in which birefringence is small and the dichroism parameter $\gamma_{a}$ is negative, giving the $x$ polarization a lower threshold. The laser begins to lase on the $x$ polarization and, if birefringence is small enough, for increasing injection current a PS occurs to the $y$ polarization, in spite of
TABLE I

PARAMETERS USED IN THE VCSEL SIMULATION

\begin{tabular}{lcr} 
Value & Parameter & Description \\
\hline $3 \mu \mathrm{m}$ & $a$ & Radius of the active region \\
$3.5,3.49$ & $n_{y}^{\text {core }}, n^{\text {clad }}$ & Refractive indices \\
3 & $\alpha$ & Linewidth enhancement factor \\
$300 \mathrm{~ns}^{-1}$ & $k$ & Field decay rate \\
$1 \mathrm{~ns}^{-1}$ & $\gamma_{N}$ & Carrier recombination rate \\
$50 \mathrm{~ns}^{-1}$ & $\gamma_{s}$ & Spin-flip rate \\
$10^{-6} \mathrm{~ns}^{-1}$ & $\beta_{s p}$ & Spontaneous emission rate
\end{tabular}

the fact that the $y$ polarization has a worst transverse confinement than the $x$ polarization and thus has less modal gain. This type of PS, that is from the low frequency (high modal gain) to the high frequency (low modal gain) polarization, has been referred to in the literature as type II [12], and has been explained, in the context of the SFM model, in terms of the interplay of birefringence, saturable dispersion, and spin-flip processes [4]. The particular value of injection current at which the PS occurs depends on the diffusion coefficient [37] and on the injection current sweep rate [38], [39]. Fig. 1(a) and (b) display the PS for slow and fast carrier diffusion and the same sweep rate (the current parameter increases linearly from $\mu_{i}=0.8$ to $\mu_{f}=1.8$ in $200 \mathrm{~ns}$ ). It can be observed that the PS is preceded by oscillations in the two polarizations.

We studied the response to a small-amplitude current modulation with a $j_{\mathrm{dc}}$ value below, near and above the PS point, for both, slow and fast carrier diffusion.

Fig. 2 displays the extreme values of the oscillations of the total intensity and of the $x$ and $y$ polarizations as a function of the modulation frequency. A clear symmetric peak, characteristic of forced oscillators, is observed below and above the switching point. The modulation frequency at which the resonance occurs is, as expected, the relaxation-oscillation frequency, $f_{\text {ro }}$, that increases with the modulation dc value. Comparing results for slow and fast carrier diffusion (left and right column of Fig. 2, respectively) it can be observed that the resonance peak is more pronounced for fast diffusion.

Near the PS there are oscillations in both polarizations which have a complex dependency on the modulation frequency. As an example, the temporal evolution of the intensities of the $x$ and $y$ modes and the total power, for fixed $j_{\mathrm{dc}}$ and $A_{m}$, and different values of $f_{m}$ are presented in Fig. 3. It is observed that the dynamics is very similar for slow and fast carrier diffusion (left and right column of Fig. 3, respectively), the only significant difference being at modulation frequencies near $f_{\text {ro }}$, where the pulses are larger for fast diffusion.

We also calculated the modulation response of the total intensity and of the $x$ and $y$ polarizations, by Fourier transforming the intensities and selecting the component of the spectrum at the modulation frequency, $\left|I_{T, x, y}\left(\nu=f_{m}\right)\right|$. Results are displayed in Fig. 4, for the same parameters as in Fig. 2. The characteristic small-signal response, with a single peak at $f_{\text {ro, }}$, is observed below and above the PS. The modulation peak is sharper for fast diffusion and for both, fast and slow diffusion, the response can be fitted by the functional form $|H(f)| \propto$ $\left[\left(f^{2}-f_{\mathrm{ro}}^{2}\right)^{2}+(\gamma f)^{2}\right]^{-1 / 2}$, where $\gamma$ is a damping factor. Near the PS there is a complex response at low frequencies that originates 

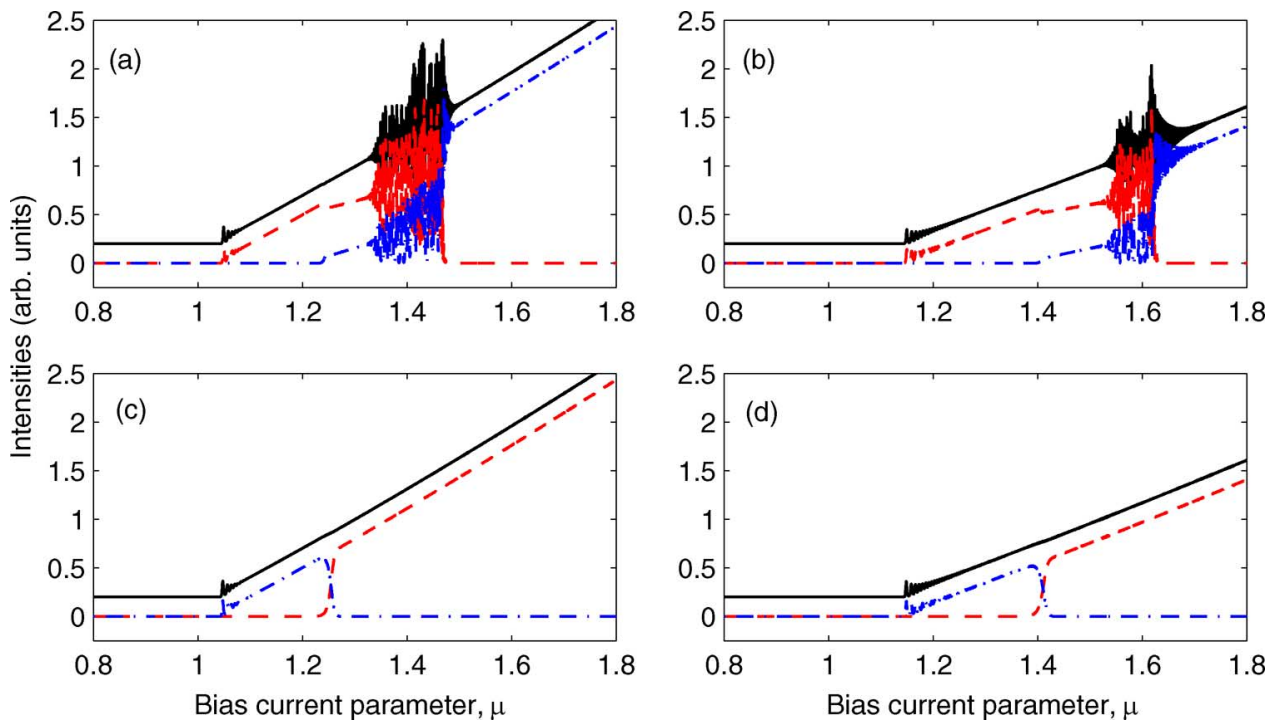

Fig. 1. (a)-(b) Type II PS occurring for negative dichroism and small birefringence. Parameters are $\gamma_{a}=-0.2 \mathrm{~ns}^{-1}$ and $n_{x}^{\text {core }}=3.50001$ (the frequency split between the two polarizations is $\gamma_{p} / \pi=1 \mathrm{GHz}$ ). (c)-(d) Type I PS occurring for positive dichroism and larger birefringence. Parameters are $\gamma_{a}=0.4$ ns ${ }^{-1}$ and $n_{x}^{\text {core }}=3.5002$ (the frequency split is $\gamma_{p} / \pi=20 \mathrm{GHz}$ ). (a) and (c) are for slow carrier diffusion, $D=0.03 \mu \mathrm{m}^{2} / \mathrm{ns}$; (b) and (d) are for faster carrier diffusion, $D=3.0 \mu \mathrm{m}^{2} / \mathrm{ns}$. The total intensity is plotted with a solid line (displaced vertically for clarity), the $x$ polarization with a dashed line, and the $y$ polarization with a dotted-dashed line.

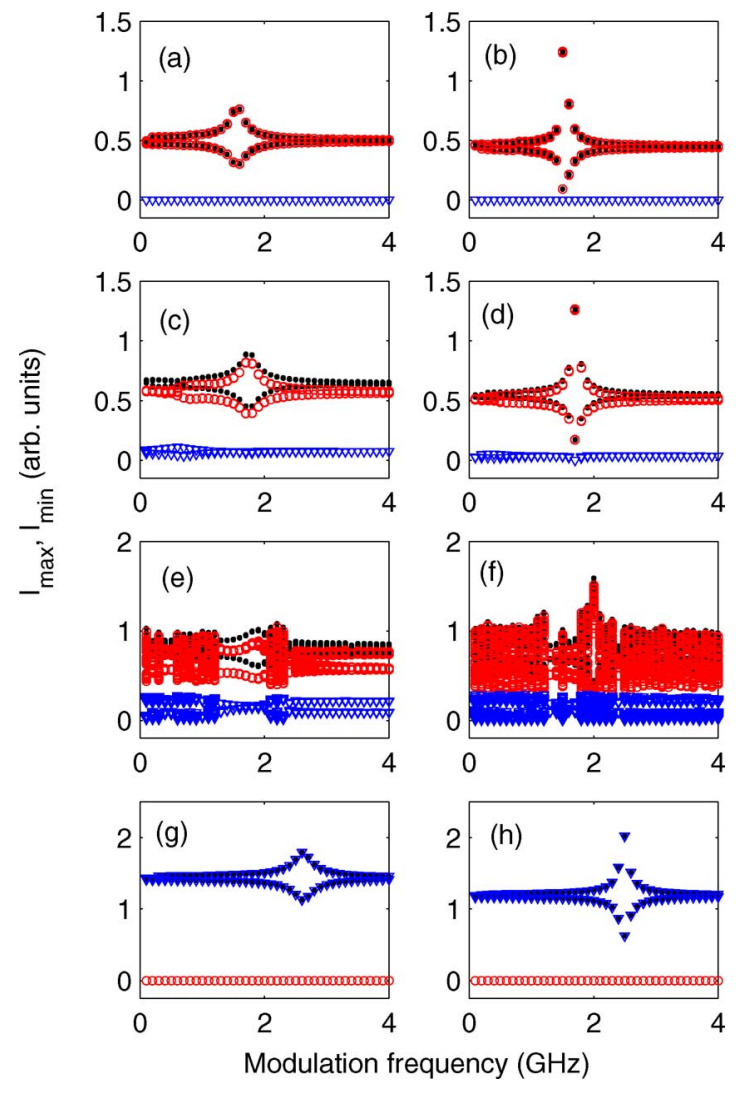

Fig. 2. Extreme values of the oscillations of the $x$ polarization (circles), the $y$ polarization (triangles), and the total intensity (dots) versus the modulation frequency for fixed modulation amplitude, $A_{m}=0.01$. Parameters correspond to the PS displayed in Fig. 1(a) and (b) and values of $j_{\mathrm{dc}}$ below, near and above the PS point. Left column: slow carrier diffusion, $D=0.03 \mu \mathrm{m}^{2} / \mathrm{ns}$, and (a) $j_{\mathrm{dc}}=1.2$, (c) 1.25 , (e) 1.3, (g) 1.5 . Right column: fast carrier diffusion, $D=3.0 \mu \mathrm{m}^{2} / \mathrm{ns}$, and (b) $j_{\mathrm{dc}}=1.35$, (d) 1.4 , (f) 1.5 , (h) 1.7 .

in polarization co-existence and competition [Fig. 4(e)-(f)]. The

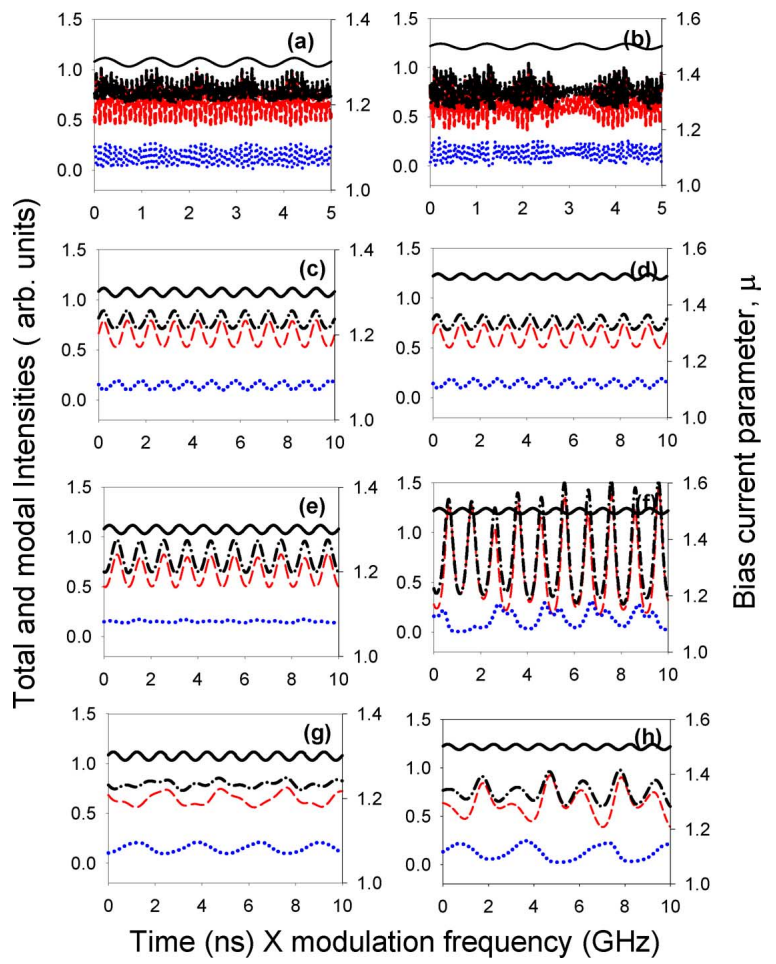

Fig. 3. Time traces of the total power (dash-dotted line), the $x$ polarization (dashed line), the $y$ polarization (dotted line), and the injection current (solid line), for various modulation frequencies and fixed modulation amplitude. The time is plotted in units of the modulation period, i.e., $t * f_{m}$. Parameters as in Fig. 2(e) and (f). Left column: slow carrier diffusion, $D=0.03 \mu \mathrm{m}^{2} / \mathrm{ns}$ and $j_{\mathrm{dc}}=1.3$, right column: fast carrier diffusion, $D=3.0 \mu \mathrm{m}^{2} / \mathrm{ns}$ and $j_{\mathrm{dc}}=1.5$. (a)-(b) $f_{m}=0.1 \mathrm{GHz}$; (c)-(d) $1.3 \mathrm{GHz}$. (e)-(f) $2.0 \mathrm{GHz}$. (g)-(h) 3.0 GHz.

small-signal modulation response of both, the $x$ polarization and the $y$ polarization is above the $-3-\mathrm{dB}$ level at low modulation 

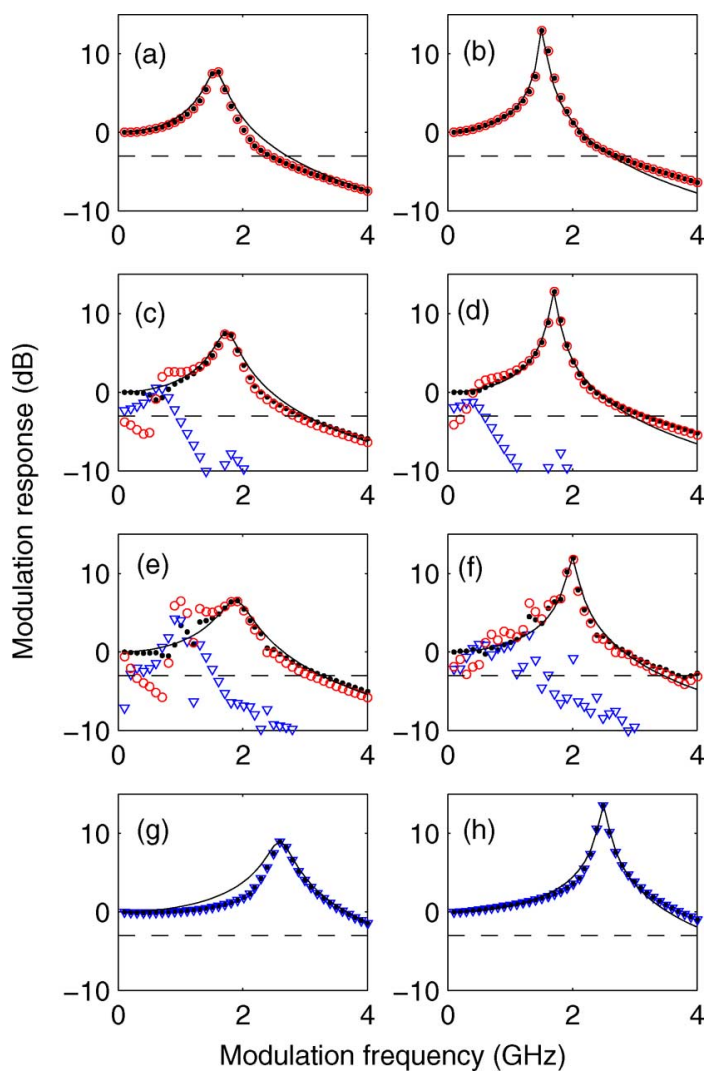

Fig. 4. Modulation response, calculated from the numerical solution in the Fourier domain. Parameters are as in Fig. 2. Total intensity (dots), $x$ polarization (circles), $y$ polarization (triangles), and fit to the single-mode small-signal modulation response, $10 \log _{10}|H(f)|$ (solid line).

frequencies, while there is single-mode response at frequencies above $f_{\text {ro. }}$.

\section{B. Small-Amplitude Modulation Near Type I PS}

Next we consider a situation in which birefringence is larger and the dichroism parameter $\gamma_{a}$ is positive, to give the $y$ polarization a lower threshold. In this situation the laser starts lasing in the $y$ polarization and as the current increases, if birefringence is large enough, at a certain point a PS occurs to the better confined $x$ polarization. This type of PS, referred to as type I [12], is displayed in Fig. 1(c) and (d), for slow and fast carrier diffusion, and the same current sweep rate as in Fig. 1(a) and (b). It can be noticed that the PS is abrupt. For injection currents near the PS point there is hysteresis, bistability and noise-induced switching. Since our goal is to study the influence of transverse effects in the polarization competition, the spontaneous emission rate and the cavity anisotropies are set to values such as to avoid noise-induced switching $\left(\beta_{s p}\right.$ is indicated in Table I and kept constant in all the simulations).

The effect of small-amplitude current modulation below and above the PS point, for slow and fast diffusion, is illustrated in Fig. 5. A symmetric peak with the same characteristics as before is observed (centered at $f_{\text {ro }}$ and sharper for fast diffusion). The small-signal numerical response in the Fourier domain of the total intensity and of the $x$ and $y$ polarizations is displayed in Fig. 6, for the same parameters as in Fig. 5. It can be noticed that, in spite of the polarization competition, the response of

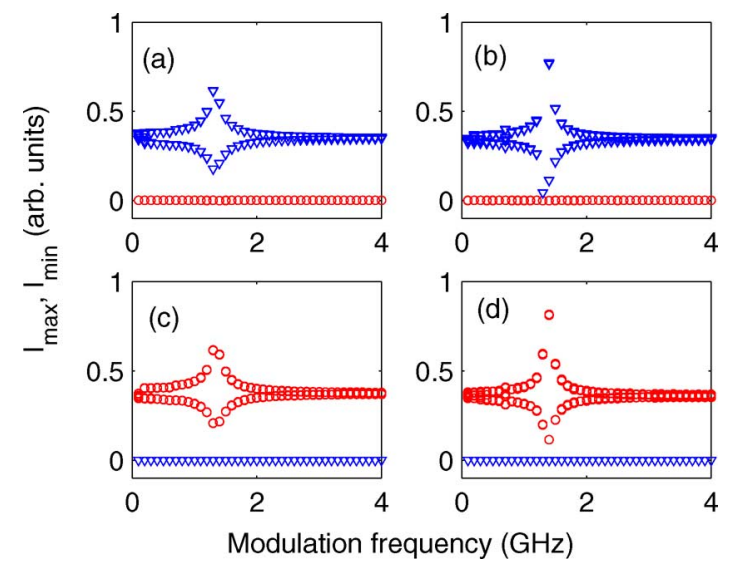

Fig. 5. Extreme values of the oscillations of the $x$ polarization (circles) and the $y$ polarization (triangles) versus the modulation frequency for fixed modulation amplitude, $A_{m}=0.01$. Parameters are as in Fig. 1(c) and (d) and $j_{\mathrm{dc}}$ is below and above the PS. Left column: slow carrier diffusion, $D=0.03 \mu \mathrm{m}^{2} / \mathrm{ns}$, and (a) $j_{\mathrm{dc}}=1.15$, (c) 1.16 . Right column: fast carrier diffusion, $D=3.0 \mu \mathrm{m}^{2} / \mathrm{ns}$, and (b) $j_{\mathrm{dc}}=$ and (d) 1.3 .

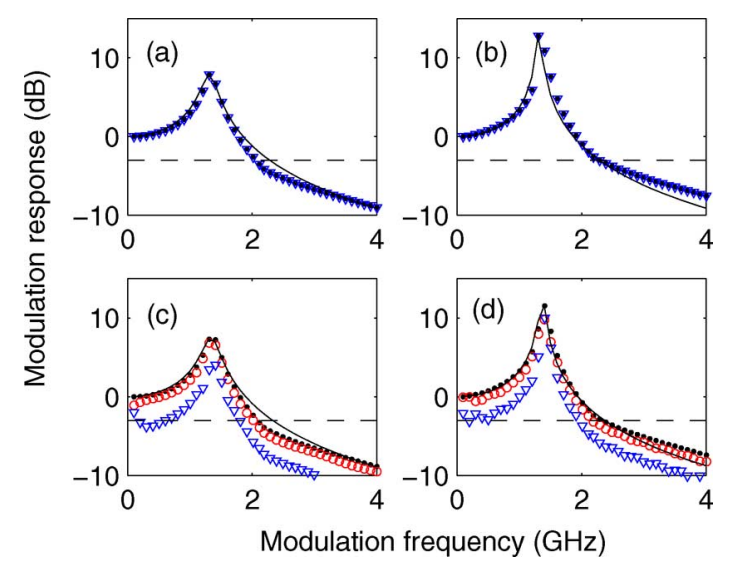

Fig. 6. Modulation response, calculated from the numerical solution in the Fourier domain, for the same parameters as in Fig. 5 (left column: slow carrier diffusion, right column: fast carrier diffusion). Total intensity (dots), $x$ polarization (circles), $y$ polarization (triangles), and fit to the single-mode small-signal modulation response, $10 \log _{10}|H(f)|$ (solid line).

the total power as well as of the two polarizations has the same functional dependency on the modulation frequency, and can be well fitted by the single-mode response function, $|H(f)|$.

In Fig. 5(a) and (c), it can be seen that the PS occurs in between $\mu=1.15$ and $\mu=1.16$, whereas in Fig. 1(c) the PS is at about $\mu=1.25$. Also, in Fig. 5(b) and (d), the PS occurs in between $\mu=1.3$ and $\mu=1.31$, whereas in Fig. 1(d) the PS is at about $\mu=1.4$. The discrepancy arises due to the fact that when the injection current increases in time (in Fig. 1 it grows from 0.8 to 1.8 in $200 \mathrm{~ns}$ ) the PS does not take place at the "static" bifurcation point (defined for $\mu$ constant in time) but occurs at a higher value, as was discussed in detail in [38], [39].

The diffusion of carriers is seen to enhance the resonance peaks in the time domain (Figs. 2 and 5) and in the frequency domain (Figs. 4 and 6). One should keep in mind, when comparing results for slow and fast diffusion, that the injection current parameter is also different: its value is such that, for low and large diffusion, we are near the PS point. Comparing the left and right columns of Figs. 2 and 5 (and also, of Figs. 4 


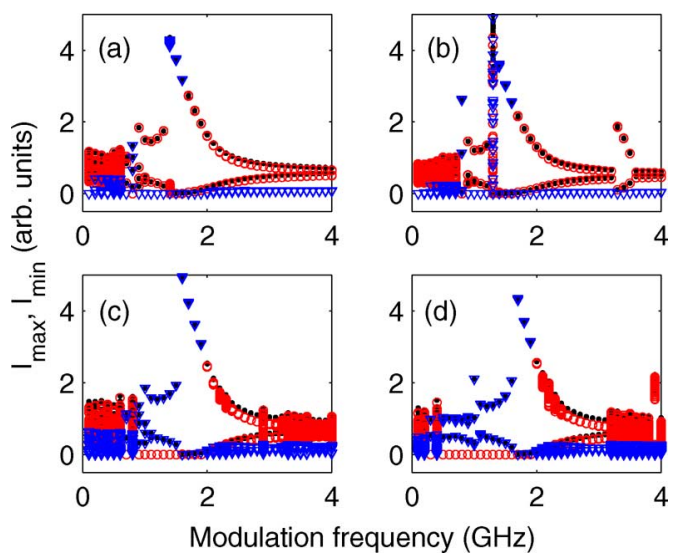

Fig. 7. Extreme values of the intensity oscillations versus the modulation frequency for parameters near type II PS and large-amplitude modulation, $A_{m}=$ 0.1 . Left column: slow carrier diffusion, $D=0.03 \mu \mathrm{m}^{2} / \mathrm{ns}$; right column: fast carrier diffusion, $D=3.0 \mu \mathrm{m}^{2} / \mathrm{ns}$. $j_{\mathrm{dc}}=$ (a) 1.25 , (b) 1.4 , (c) 1.3 , (d) 1.5 .

and 6), it can be seen that the current effectively injected into the active region of the laser is about the same for large and low $\mathrm{D}$, because the output intensity in the absence of modulation is the same (e.g., in Fig. 2(a) and (b), the continuous-wave (CW) output intensity is 0.5 for both, $D=0.03 \mu \mathrm{m}^{2} / \mathrm{ns}$ and $D=3 \mu \mathrm{m}^{2} / \mathrm{ns}$ ), and the frequency of the relaxation oscillations is the same for $D=0.03 \mu \mathrm{m}^{2} / \mathrm{ns}$ and $D=3 \mu \mathrm{m}^{2} / \mathrm{ns}$ [e.g., in Fig. 2(a) and (b), the resonance peak occurs at the same frequency of about $1.5 \mathrm{GHz}$, but the peak is sharper and more pronounced for $\left.D=3 \mu \mathrm{m}^{2} / \mathrm{ns}\right)$.

\section{Larger Amplitude Modulation}

Figs. 7 and 8 display results for larger modulation amplitude, low and large birefringence, and values of $j_{\mathrm{dc}}$ near type II and type I PS, respectively $\left(A_{m}=0.1\right.$, other parameters are as in Figs. 2 and 5). The plot of the extreme values of the intensity oscillations reveals a distortion of the resonance peak that is asymmetric, suggesting the coexistence of solutions with different amplitude and the same frequency, e.g., large pulses and smaller sinusoidal oscillations. The plot of the extreme values of the total intensity is very similar for low and large birefringence, indicating that polarization competition does not have a significant impact on the oscillations of the total power, that are governed by the current modulation. Similar asymmetric curves have been found previously in the literature, using models that do not take into account either transverse or polarization effects [40], [41].

Comparing results for slow and fast diffusion (left and right columns of Figs. 7 and 8, respectively) we notice also that carrier diffusion does not have a significant impact, the main difference being a resonance at modulation frequencies near $2 f_{\text {ro }}$ ( $\sim 3 \mathrm{GHz}$ ), that is more pronounced for large $D$.

In Fig. 7 it can be observed that the polarization state of the light varies with the modulation frequency: for slow and fast modulation there is polarization coexistence, while for modulation frequencies near $f_{\text {ro }}$ there is single-polarization emission (the $x$ polarization is off). In this parameter region we have observed that there is multi-stability of solutions and the polarization depends not only on the modulation frequency but

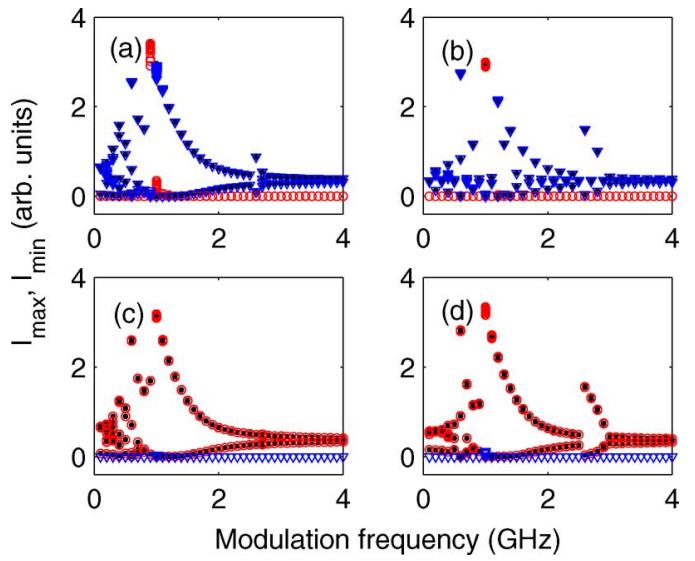

Fig. 8. Extreme values of the intensity oscillations versus the modulation frequency for parameters near type I PS and large-amplitude modulation, $A_{m}=$ 0.1 . Left column: slow carrier diffusion, $D=0.03 \mu \mathrm{m}^{2} / \mathrm{ns}$; right column: fast carrier diffusion, $D=3.0 \mu \mathrm{m}^{2} / \mathrm{ns}$. $j_{\mathrm{dc}}=$ (a) 1.15 , (b) 1.3 , (c) 1.16 , (d) 1.31 .

also on the way the injection current is varied. Fig. 9 displays an example: in Fig. 9(a) the modulation starts after a transient in which $j(t)$ is constant (and equal to the modulation dc value); in Fig. 9(b)-(d), the modulation starts after a transient in which $j(t)$ increases gradually to the modulation dc value, and is observed that the polarization varies with the modulation frequency: there is oscillation on both polarizations for $f_{m}=4 \mathrm{GHz}$ [Fig. 9(b)] and $f_{m}=0.4 \mathrm{GHz}$ [Fig. 9(d)], but only on the $y$ polarization for $f_{m}=1.5 \mathrm{GHz}$ [Fig. 9(c)].

The plots of the extreme oscillation values displayed in Figs. 7 and 8 provide us with information about the relative amplitude of the two polarizations, but not about the phase relation between their oscillations. An inspection of the time traces reveals inphase pulses when the modulation is fast, while for slow modulation, the inphase pulses are accompanied by a slower out-of-phase behavior. As an example, a few temporal evolutions are presented in Fig. 10, for various modulation frequencies and fixed values of $A_{m}$ and $j_{\mathrm{dc}}$. For fast modulation the $y$ polarization dominates and exhibits large pulses, that are accompanied by smaller pulses of the $x$ polarization [Fig. 10(a) and (b)]. As the modulation becomes slower, the pulses turn into trains of pulses and their amplitude decrease [Fig. 10(c) and (d)]. For slow enough modulation the trains of pulses become a repetition of turn-on and turn-off events [Fig. 10(e)]. Out of phase behavior can be observed because in some time intervals the $x$ polarization dominates, and in others, the $y$ polarization dominates.

Results of simulations of the original SFM equations (without spatial effects) presented in [34] show a similar influence of the modulation frequency (see [34, Fig. 3]): the amplitude of the intensity oscillations decreases as the modulation becomes faster, and the polarization of the light varies with the modulation frequency. The comparison with [33] is not so straightforward because in [33] several transverse modes are considered (LP01, LP11c, LP11s, LP02, etc.) and the authors study the bifurcations in terms of a control parameter that is the dc value of the current modulation. Nevertheless, multistability of solutions is found, as we also do, for large modulation amplitude. 


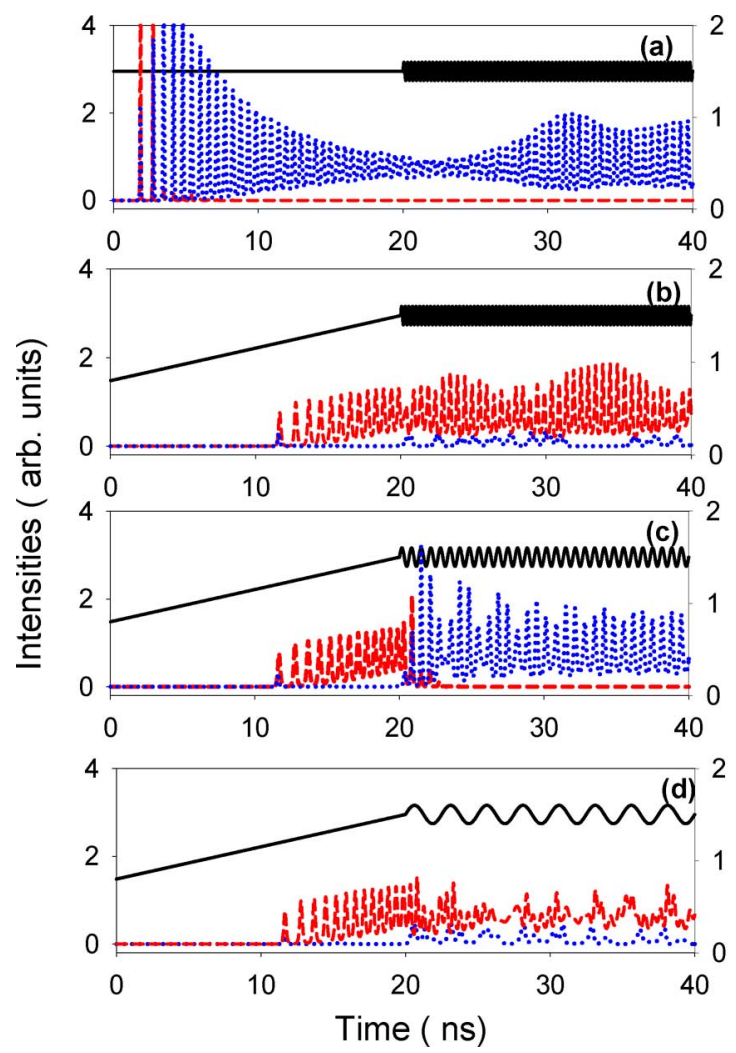

Fig. 9. Time traces of the $x$ polarization (dashed line), the $y$ polarization (dotted line), and the injection current (solid line) when the modulation starts after a transient with constant injection current (a), and after a transient in which the injection current increases gradually (b)-(d). The modulation frequency is: (a) and (b) $f_{m}=4 \mathrm{GHz}$, (c) $1.5 \mathrm{GHz}$, and (d) $0.4 \mathrm{GHz}$ (d). Other parameters are as in Fig. 7(d).

\section{SUMMARY AND CONCLUSION}

We studied numerically the dynamics of current-modulated VCSELs within the framework of a spatially dependent spin-flip model. The fundamental $L P_{01}$ transverse mode of a cylindrical waveguide was used to describe the transverse profile of two orthogonal linear polarizations. Birefringence was taken into account by considering different core refractive indexes for the two polarizations. For low birefringence we studied the influence of current modulation near the PS of type II, from the low to the high frequency polarization; for larger birefringence we studied the influence of current modulation near the PS of type $\mathrm{I}$, from the high to the low frequency polarization.

The influence of the bias dc value, the modulation amplitude, and the modulation frequency were analyzed. Different dynamic regimes, including polarization competition and polarization coexistence were found. For small modulation amplitude there are oscillations of the total intensity that are enhanced at modulation frequencies close to the relaxation oscillation frequency $f_{\text {ro. }}$. A plot of the extreme values of the oscillations versus the modulation frequency reveals a resonance curve with a maximum near the relaxation oscillation frequency, and the characteristic shape of forced nonlinear oscillators. The influence of carrier diffusion is mainly an enhancement of the resonance peak at frequencies close to $f_{\mathrm{ro}}$. We interpret this in the following way: at fast modulation frequencies, the response of the laser decreases rapidly, and the diffusion of carriers plays no

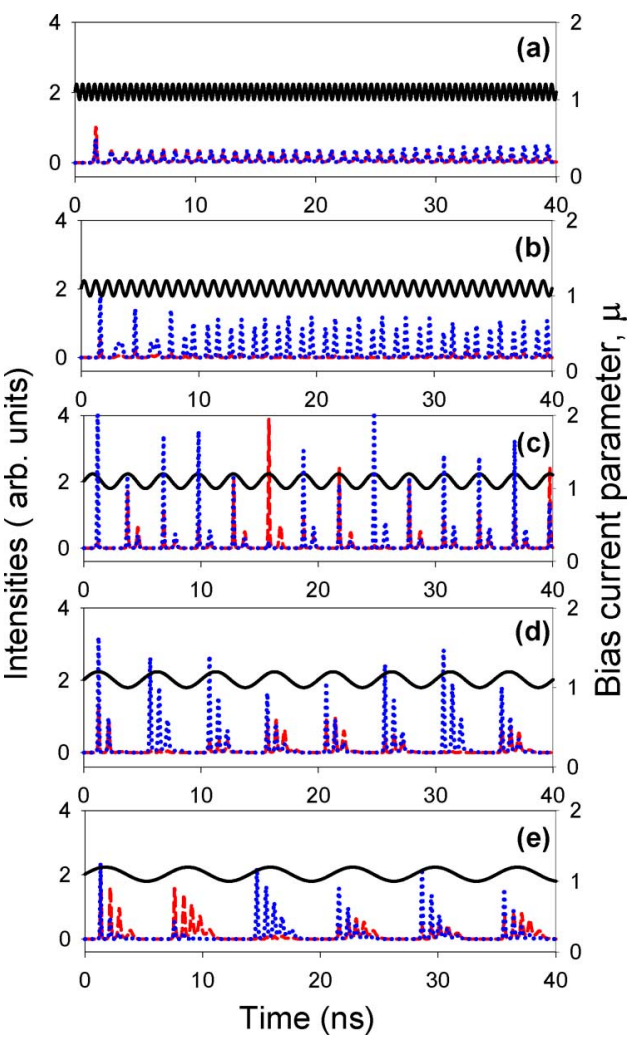

Fig. 10. Time traces of the $x$ and $y$ polarizations, for fixed modulation amplitude, $A_{m}=0.1$, and various modulation frequencies: (a) $f_{m}=2 \mathrm{GHz}$, (b) $1 \mathrm{GHz}$, (c) $0.33 \mathrm{GHz}$, (d) $0.2 \mathrm{GHz}$, and (e) $0.143 \mathrm{GHz}$. Other parameters are: $j_{\mathrm{dc}}=1.1, \eta_{x}^{\text {core }}=3.5002, \gamma_{a}=0.3 \mathrm{~ns}^{-1}$, and $D=0.03 \mu \mathrm{m}^{2} / \mathrm{ns}$.

role in the response because the modulation of the injected current is so fast that the carriers do not have time to diffuse; at low modulation frequencies, we are in the complementary situation of a quasi-static regime in which the current modulation is slow enough to permit the carriers to diffuse. For modulation frequencies near $f_{\text {ro }}$, fast carrier diffusion enhances the amplitude of the intensity oscillations because of the spatial hole burning effect: the faster the diffusion, the faster the carriers move to fill up the spatial hole burned by the growing intensity, thus allowing for larger intensity pulses (which translate, in the frequency domain, to a more pronounced peak in the modulation response, at modulation frequencies near $f_{\text {ro }}$ ).

We characterized the small-signal polarization-resolved modulation response in the Fourier domain for bias dc values below, near and above the polarization switching point. Close to type I PS the modulation response of the total power as well as the modulation response of the two orthogonal polarizations has the same functional dependency on the modulation frequency and can be fitted by the response function of a single-mode laser. Close to type II PS, polarization competition is a significant process at modulation frequencies near and below the relaxation oscillation frequency, because the intrinsic polarization oscillations that precede the PS (these oscillations are not induced by the current modulation) have a lower frequency than the relaxation oscillations.

The main conclusion of our work, which has been performed using parameters that are typical for VCSELs, is that carrier diffusion and transverse inhomogeneities have little 
influence on the polarization competition, which is mainly determined by the same physical mechanisms as in the original SFM model, i.e,. the combined nonlinear interplay of cavity anisotropies (dichroism and birefringence), saturable dispersion (alpha-factor) and the spin-flip relaxation rate [4]. We consider that our work can provide a connection between measured VCSEL data (such as the modulation response) and the SFM model parameters, that are difficult to estimate directly, but they can be globally adjusted such as to fit both the resonance frequency and the resonance linewidth.

In spite of the polarization competition, the modulation response of the total power can be fitted by the single-mode laser response function. The modulation response of the orthogonal polarizations displays features at low frequencies that are not present in the response of the total power. In principle, it would be possible to fit the modulation response to a theoretical curve, following the same procedure as in [42], where the relative intensity noise of multimode VCSELs was calculated analytically.

To the best of our knowledge, this is the first study of the modulation response of VCSELs based on the SFM model. Further investigations to clarify the role of spin-flip relaxation and spontaneous emission noise will be very interesting .However, due to the fact that spatial effects have little influence on the polarization competition, the study of the role of the spin-flip rate and the noise level can be done based the original SFM model ( thus obviating the computational overheads incurred when account is taken of spatial effects).

For larger modulation amplitude we found complex pulsing behavior, with the orthogonal polarizations exhibiting a rich variety of oscillation regimes depending on the modulation frequency. We hope that these results will motivate new experiments on the polarization dynamics of current modulated VCSELs. A pronounced resonance peak can be desirable for applications in which the laser operates at a well-defined modulation frequency. We suppose that by the use of appropriate doping profiles the carrier diffusion coefficient can be carefully calibrated (within a limited interval), thus allowing the design of devices that yield the desired resonance at the specified modulation rate.

\section{REFERENCES}

[1] Z. G. Pan, S. J. Jiang, M. Dagenais, R. A. Morgan, K. Kojima, M. T. Asom, R. E. Leibenguth, G. D. Guth, and M. W. Focht, "Optical-injection induced polarization bistability in vertical-cavity surface-emitting lasers," Appl. Phys. Lett., vol. 63, pp. 2999-3001, 1993.

[2] K. D. Choquette, R. P. Schneider, K. L. Lear, and R. E. Leibenguth, "Gain-dependent polarization properties of vertical-cavity lasers," IEEE J. Sel. Topics Quantum Electron., vol. 1, no. 6, pp. 661-666, Jun. 1995.

[3] A. Valle, J. Sarma, and K. A. Shore, "Spatial holeburning effects on the dynamics of vertical-cavity surface-emitting laser-diodes," IEEE J. Quantum Electron., vol. 31, no. 8, pp. 1423-1431, Aug. 1995.

[4] J. Martin-Regalado, F. Prati, M. S. Miguel, and N. B. Abraham, "Polarization properties of vertical-cavity surface-emitting lasers," IEEE J. Quantum Electron., vol. 33, no. 5, pp. 765-783, May 1997.

[5] J. Martin-Regalado, S. Balle, M. S. Miguel, A. Valle, and L. Pesquera, "Polarization and transverse-mode selection in quantum-well vertical-cavity surface-emitting lasers: Index- and gain-guided devices," Quantum Semiclass. Opt., vol. 9, pp. 713-736, 1997.

[6] A. Valle, L. L. Pesquera, and K. A. Shore, "Polarization behavior of birefringent multitransverse mode vertical-cavity surface-emitting lasers," IEEE Photon. Technol. Lett., vol. 9, no. 5, pp. 557-559, May 1997.
[7] M. Travagnin, M. P. van Exter, and J. P. Woerdman, "Influence of carrier dynamics on the polarization stability and noise-induced polarization hopping in surface-emitting semiconductor lasers," Phys. Rev. A, vol. 56, pp. 1497-1507, 1997.

[8] G. Giacomelli and F. Marin, "Statistics of polarization competition in VCSELs," Quantum Semiclass. Opt., vol. 10, pp. 469-476, 1998.

[9] K. Panajotov, B. Ryvkin, J. Danckaert, M. Peeters, H. Thienpont, and I. Veretennicoff, "Polarization switching in VCSEL's due to thermal lensing," IEEE Photon. Technol. Lett., vol. 10, no. 1, pp. 6-8, Jan. 1998.

[10] M. B. Willemsen, M. U. F. Khalid, M. P. van Exter, and J. P. Woerdman, "Polarization switching of a vertical-cavity semiconductor laser as a Kramers hopping problem," Phys. Rev. Lett., vol. 82, pp. 4815-4818, 1999.

[11] S. Balle, E. Tolkachova, M. S. Miguel, J. R. Tredicce, J. Martin-Regalado, and A. Gahl, "Mechanisms of polarization switching in single-transverse-mode vertical-cavity surface-emitting lasers: Thermal shift and nonlinear semiconductor dynamics," Opt. Lett., vol. 24, pp. 1121-1123, 1999.

[12] B. Ryvkin, K. Panajotov, A. Georgievski, J. Danckaert, M. Peeters, G. Verschaffelt, H. Thienpont, and I. Veretennicoff, "Effect of photonenergy-dependent loss and gain mechanisms on polarization switching in vertical-cavity surface-emitting lasers," J. Opt. Soc. Amer. B, vol. 16, pp. 2106-2113, 1999.

[13] T. Ackemann and Sondermann, "Characteristics of polarization switching from the low to the high frequency mode in vertical-cavity surface-emitting lasers," Appl. Phys. Lett., vol. 78, pp. 3574-3576, 2001.

[14] Y. Hong, K. A. Shore, A. Larsson, M. Ghisoni, and J. Halonen, "Polarisation switching in a vertical cavity surface emitting semiconductor laser by frequency detuning," Proc. IEE Optoelectron., vol. 148, pp. 31-34, 2001.

[15] "Special Issue on polarization effects in fiber-optic networks," $J$. Lightw. Technol., vol. 24, no. 11, Nov. 2006.

[16] K. D. Choquette and R. E. Leibenguth, "Control of vertical-cavity laser polarization with anisotropic transverse cavity geometries," IEEE Photon. Technol. Lett., vol. 6, no. 1, pp. 40-42, Jan. 1994.

[17] T. H. Russell and T. D. Milster, "Polarization switching control in vertical-cavity surface-emitting lasers," Appl. Phys. Lett., vol. 70, pp. 2520-2522, 1997.

[18] Y. H. Hong, P. S. Spencer, and K. A. Shore, "Suppression of polarization switching in vertical-cavity surface-emitting lasers by use of optical feedback," Opt. Lett., vol. 29, pp. 2151-2153, 2004.

[19] J. M. Ostermann, P. Debernardi, C. Jalics, and R. Michalzik, "Polarization-stable oxide-confined VCSELs with enhanced single-mode output power via monolithicaily integrated inverted grating reliefs," IEEE J. Sel. Topics Quantum Electron., vol. 11, no. 5, pp. 982-989, Sep./Oct. 2005.

[20] K. L. Lear, A. Mar, K. D. Choquette, S. P. Kilcoyne, R. P. Schneider, and K. M. Geib, "High-frequency modulation of oxide-confined vertical cavity surface emitting lasers," Electron. Lett., vol. 32, pp. 457-458, Feb. 1996.

[21] S. F. Yu, "Dynamic behavior of vertical-cavity surface-emitting lasers," IEEE J. Quantum Electron., vol. 32, no. 7, pp. 1168-1179, Jul. 1996.

[22] B. J. Thibeault, K. Bertilsson, E. R. Hegblom, E. Strzelecka, P. D. Floyd, R. Naone, and L. A. Coldren, "High-speed characteristics of low-optical loss oxide-apertured vertical-cavity lasers," IEEE Photon. Technol. Lett., vol. 9, no. 1, pp. 11-13, Jan. 1997.

[23] J. Y. Law and G. P. Agrawal, "Effects of spatial hole burning on gain switching in vertical-cavity surface-emitting lasers," IEEE J. Quantum Electron., vol. 33, no. 3, pp. 462-468, Mar. 1997.

[24] Y. Satuby and M. Orenstein, "Small-signal modulation of multitransverse modes vertical-cavity surface-emitting semiconductor lasers," IEEE Photon. Technol. Lett., vol. 10, no. 6, pp. 757-759, Jun. 1998.

[25] M. S. Torre and H. F. Ranea-Sandoval, "Modulation response of multiple transverse modes in vertical-cavity surface-emitting lasers," IEEE J. Quantum Electron., vol. 36, no. 1, pp. 112-117, Jan. 2000.

[26] J. S. Gustavsson, A. Haglund, J. Bengtsson, and A. Larsson, "High-speed digital modulation characteristics of oxide-confined vertical-cavity surface-emitting lasers - Numerical simulations consistent with experimental results," IEEE J. Quantum Electron., vol. 38, no. 8, pp. 1089-1096, Aug. 2002.

[27] Y. Liu, W. C. Ng, B. Klein, and K. Hess, "Effects of the spatial nonuniformity of optical transverse modes on the modulation response of vertical-cavity surface-emitting lasers," IEEE J. Quantum Electron., vol. 39, no. 1, pp. 99-108, Jan. 2003.

[28] C. H. Chang, L. Chrostowski, and C. J. Chang-Hasnain, "Injection locking of VCSELs," IEEE J. Sel. Topics Quantum Electron., vol. 9 , no. 5, pp. 1386-1393, Sep./Oct. 2003. 
[29] A. Valle and L. Pesquera, "Analysis of bit-error rate of vertical-cavity surface-emitting lasers modulated at high speed," IEEE J. Quantum Electron., vol. 42, no. 3, pp. 435-446, Mar./Apr. 2006.

[30] D. M. Grasso, D. K. Serkland, G. M. Peake, K. M. Geib, and K. D. Choquette, "Direct modulation characteristics of composite resonator vertical-cavity lasers," IEEE J. Quantum Electron., vol. 42, no. 6, pp. 1248-1254, Nov./Dec. 2006.

[31] G. Verschaffelt, J. Albert, I. Veretennicoff, J. Danckaert, S. Barbay, G. Giacomelli, and F. Marin, "Frequency response of current-driven polarization modulation in vertical-cavity surface-emitting lasers," Appl. Phys. Lett., vol. 80, pp. 2248-2250, 2002.

[32] G. Verschaffelt, J. Albert, B. Nagler, M. Peeters, J. Danckaert, S. Barbay, G. Giacomelli, and F. Marin, "Frequency response of polarization switching in vertical-cavity surface-emitting lasers," IEEE $J$. Quantum Electron., vol. 39, no. 10, pp. 1177-1186, Oct. 2003.

[33] A. Valle, L. Pesquera, S. I. Turovets, and J. M. Lopez, "Nonlinear dynamics of current-modulated vertical-cavity surface-emitting lasers," Opt. Communs., vol. 208, pp. 173-182, 2002.

[34] M. Sciamanna, A. Valle, P. Megret, M. Blondel, and K. Panajotov, "Nonlinear polarization dynamics in directly modulated vertical-cavity surface-emitting lasers," Phys. Rev. E, vol. 68, no. 016207, 2003.

[35] M. S. Torre, C. Masoller, P. Mandel, and K. A. Shore, "Transversemode dynamics in directly modulated vertical-cavity surface-emitting lasers with optical feedback," IEEE J. Quantum Electron., vol. 40, no. 6, pp. 620-628, Jun. 2004.

[36] Y. H. Hong, S. Bandyopadhyay, and K. A. Shore, "Spectral signatures of the dynamics of current-modulated vertical-cavity surface-emitting lasers subject to optical feedback," J. Opt. Soc. Amer. B, vol. 22, pp. 2350-2356, 2005

[37] M. S. Torre, C. Masoller, and P. Mandel, "Transverse and polarization effects in index-guided vertical-cavity surface-emitting lasers," Phys. Rev. A, vol. 74, no. 043808, 2006.

[38] C. Masoller, M. S. Torre, and P. Mandel, "Influence of the injection current sweep rate on the polarization switching of vertical-cavity surface-emitting lasers," J. Appl. Phys., vol. 99, no. 026106, 2006.

[39] J. Paul, C. Masoller, Y. Hong, P. S. Spencer, and K. A. Shore, "Experimental study of polarization switching of vertical-cavity surface-emitting lasers as a dynamical bifurcation,” Opt. Lett., vol. 31, pp. 748-750, 2006.

[40] M. S. Torre, C. Masoller, P. Mandel, and K. A. Shore, "Enhanced sensitivity to current modulation near dynamic instability in semiconductor lasers with optical feedback and optical injection," J. Opt. Soc. Amer. $B$, vol. 21, pp. 302-306, 2004.

[41] B. F. Kuntsevich, A. N. Pisarchik, and V. K. Kononenko, "Nonlinear dynamics of a directly modulated semiconductor laser with cavity detuning," Opt. Quantum. Electron., vol. 37, pp. 675-693, 2005.

[42] A. Valle and L. Pesquera, "Theoretical calculation of relative intensity noise of multimode vertical-cavity surface-emitting lasers," IEEE $J$. Quantum Electron., vol. 40, pp. 597-606, Jun. 2004.

Cristina Masoller was born in Montevideo, Uruguay, in 1963. She received the M.Sc. degree in physics from the Universidad de la Republica, Montevideo, Uruguay, in 1991 and the Ph.D. degree in physics from Bryn Mawr College, Bryn Mawr, PA, in 1999.

Since 2004, she has been a Ramon and Cajal Researcher at the Universitat Politecnica de Cataluña, Cataluña, Spain. Her research interests include theoretical modeling of nonlinear dynamics of lasers, synchronization and stochastic phenomena, and time-delayed systems.
Maria Susana Torre received the degree of Licenciada en Fisica and the Ph.D degree from the Universidad Nacional del Centro de la Provincia de Buenos Aires (UNCPBA), Buenos Aires, Argentina. Her research was primarily in external driven laser physics.

From 1995 to 1997, she was a Postdoctoral Fellow at the Photonics Technology Department, ETSI Telecomunicaciones, Universidad Politécnica de Madrid. Her research was in quantum-well semiconductor laser modeling. Since 1988, she has been a member of the Quantum Electronic Group of the Physics Institute "Arroyo Seco." She is currently Research Professor at the Facultad de Ciencias Exactas of UNCPBA. Her research interests include modeling and dynamics of VCSEL, dynamics of semiconductor laser with external optical feedback and diffusion effects in semiconductor lasers.

K. Alan Shore (M'88-SM'95) received the degree in mathematics from the University of Oxford, Oxford, U.K., and the Ph.D. degree at University College, Cardiff, Wales, U.K. His thesis work was concerned with the electrical and optical properties of double-heterostructure semiconductor lasers.

He was a Lecturer at the University of Liverpool (1979-1983), Liverpool, U.K., and then at the University of Bath, Bath, U.K., where he became Senior Lecturer (1986), Reader (1990), and Professor (1995). In 1995, he was appointed to the Chair of Electronic Engineering, University of Wales, Bangor, U.K., where he is currently the Head of the School of Informatics. He is the Director of Industrial and Commercial Optoelectronics (ICON) a Welsh Development Agency Centre of Excellence which has a mission to "make light work" through the utilization of Bangor optoelectronics expertise and facilities. He is also the Deputy Chair of the Welsh Optoelectronics Forum. His research work has been principally in the area of semiconductor optoelectronic device design and experimental characterization with particular emphasis on nonlinearities in laser diodes and semiconductor optical waveguides. He has authored or co-authored almost 700 contributions to archival journals, books and technical conferences. He was a Visiting Researcher at the Center for High Technology Materials, University of New Mexico, Albuquerque, in 1987. He received a Royal Society Travel Grant to visit universities and laboratories in Japan in July 1988. In 1989, he was a Visiting Researcher at the Huygens Laboratory, Leiden University, The Netherlands. During the summers of 1990 and 1991 he worked at the Teledanmark Research Laboratory and the MIDIT Center of the Technical University of Denmark, Lyngby. He was a Guest Researcher at the Electrotechnical Laboratory (ETL), Tsukuba, Japan in 1991. In 1992, he was a Visiting Professor at the Department of Physics, University de les Illes Balears, Palma-Mallorca, Spain. He was a Visiting Lecturer in the Instituto de Fisica de Cantabria, Santander, Spain, in June 1996 and 1998, and a Visiting Researcher in the Centre for Laser Applications, Department of Physics, Macquarie University, Sydney, Australia, in July-August 1996, 1998, 2000 and April 2002. In July-August 2001, he was a Visiting Researcher at the ATR Adaptive Communications Laboratories, Kyoto, Japan. His current research interests include multiwave mixing and optical switching in semiconductor lasers, design and fabrication of intersubband semiconductor lasers and organic semiconductor lasers, dynamics of vertical-cavity semiconductor lasers and applications of nonlinear dynamics in semiconductor lasers to optical data encryption.

Dr. Shore co-founded and acts as Organizer and Programme Committee Chair for the International Conference on Semiconductor and Integrated Optoelectronics (SIOE) which, since 1987, has been held annually in Cardiff, Wales, U.K. He was Programme Chair for the U.K. National Quantum Electronic Conference (QE'13) and was European Liaison Committee chair for the OSA Integrated Photonics Research (IPR'98) conference in Victoria BC, Canada, 1998. He was a member of the programme committee for IPR'99 Santa Barbara, California, July, 1999, and also the European Conference on Integrated Optics (ECIO) Torino, Italy, in April 1999. He was a co-organizer of a Rank Prize Symposium on Nonlinear Dynamics in Lasers held in the Lake District, U.K. in August 2002. He was a technical committee member for the Conference on Lasers and Electro-Optics (CLEO), Europe, 2003. 